\title{
¿EXISTEN RELACIONES ENTRE LA FORMACIÓN CIUDADANA Y LA JUSTICIA SOCIAL EN LA EDUCACIÓN?!
}

\section{ARE THERE RELAIIONS BETWEEN CIVIC EDUCATION AND SOCIAL JUSTICE IN EDUCAIION?}

Sandra Mejīa Rodríguez, Ruth Elena Quiroz, y Ana Elsy Díaz?

RESUMEN Este artículo tiene el propósito de presentar, en términos teóricos, las relaciones conceptuales entre dos nociones, el de formación ciudadana y el de la justicia social en la educación con apoyo en la investigación "Formación ciudadana y justicia social en la educación desde la teoría del reconocimiento: discursos sobre las prácticas de los líderes del proyecto colegios maestros de Medellin", dada en el marco de la línea doctoral en Educación de Ciencias Sociales y Humanas de la Facultad de Educación de la Universidad de Antioquia. La lectura de las relaciones entre estos conceptos citados se realizó a la luz de la Teoría del Reconocimiento de Axel Honneth.

Se encontró que existe un vinculo entre las categorias del amor, el derecho y la solidaridad, categorias con las que su autor lee la justicia social y la escuela, asumida como ese espacio donde aparecen discursos y prácticas particulares, contextuales y universales que afectan de manera positiva o negativa al sujeto social, potenciando o haciendo daño en la autoconfianza, el autorrespeto y la autoestima que son formas del menosprecio y menoscaban el sujeto político.

${ }^{1}$ Artículo recibido el 28 de marzo de 2018. Aceptado el 31 de marzo de 2019.

${ }^{2}$ Doctora en Educación. Profesora de Cátedra Universidad de Antioquia, Facultad de Educación. Programa de Maestría en Educación. Correo electrónico: sandramejiarodriguez@gmail.com.

Doctora en Ciencias Pedagógicas. Profesora Titular, Universidad de Antioquia. Facultad de Educación, Programa de Doctorado en Educación. Correo electrónico: ruth.quiroz@udea.edu.co

Doctora en Educación. Profesora Titular, Universidad de Antioquia. Escuela de ldiomas. Coordinadora del grupo de investigación Pedagogía y Didáctica de las Lenguas Extranjeras. Correo electrónico: ana.diaz@ udea.edu.co

\section{INTRODUCCIÓN}

La educación que se requiere hoy debe responder, desde la formación ciudadana, a la solución de problemas cotidianos, expresados muchas veces en la vulneración de los derechos humanos, el irrespeto a la vida y el medio ambiente, las múltiples violencias y la negación de las minorias culturales, así como "la pobreza, el hambre, el desempleo, la falta de educación, la explotación, la marginalización" (Cortés, 2007: 13). Sobre tal panorama, la justicia social en la educación puede ser asumida como la búsqueda y concreción de los derechos sociales, especificamente en la necesidad de examinar la redistribución y el reconocimiento (Fraser y Honneth, 2006) de los derechos de las clases sociales, más allá de la repartición de riqueza, para instaurarse en el desarrollo de comunidades más equitativas, con mejores condiciones para todos los ciudadanos. En estos términos, en la educación entendida como proceso y como resultado de la aculturación social, se presenta la necesidad del reconocimiento de los derechos, asunto que ha trascendido a las lógicas redistributivas, centrándose cada día más en aspectos curriculares, inclusión, espacio escolar y aprendizaje contextual pertinente para formar ciudadanos que procuren la realización de derechos. Esto lleva consigo el compromiso de reflexionar con rigor, desde la investigación, las relaciones entre formación ciudadana y justicia social en la educación, específicamente desde la Teoría del Reconocimiento, para lo cual, metodológicamente hablando, se propuso describir e interpretar las narrativas sobre las prácticas de los líderes del proyecto Colegios Maestros de la ciudad de Medellín. De los resultados de la investigación en mención, en este artículo se presentará únicamente el aporte teórico de la misma, haciendo evidente la relación planteada entre la formación ciudadana y la justicia social en la educación desde la Teoría del Reconocimiento.

\section{EDUCACIÓN, FORMACIÓN CIUDADANA Y SU RESULTADO: EL EJERCICIO DE LA CIUDADANÍA}

La educación como sistema social que genera relaciones con otras instituciones sociales tiene el propósito de preparar una 
De Prácticas y discursos

Universidad Nacional del Nordeste

Centro de Estudios Sociales

Año 8, Número 11, 2019, Octubre

ISSN 2250-6942

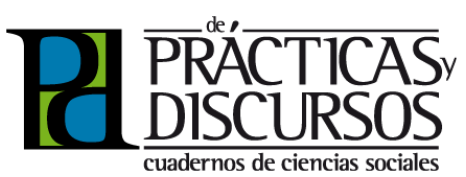

ABSTRACT This paper aims at showing theoretically the relations between two concepts:

civic education and social justice in education,

supporting it in the research named 'Formación

Ciudadana y Justicia Social en la Educación des-

de la Teoria del Reconocimiento: discursos sobre

las prácticas de los líderes del Proyecto Colegios

Maestros de Medellin/ Civic Education and Social

Justice in Education from Recognition Theory:

Discourses on Work by leaders of Colegios Maes-

tros Medellin Project'. The research is given un-

der the Doctorate Education in Social and Human

Sciences, Faculty of Education at Universidad de

Antioquia (2017). The analysis between the two

concepts was carried out in light of recognition

theory by Axel Honneth.

It was found that in effect there is a relation

between the categories Love, Right and Solida-

rity -the author uses them to understand the

social justice- and education in general terms.

Likewise, school is seen on the one hand as a

space where social justice is performed; on the

other hand, as a space where discourses and

particular, situational and universal practices

are given. These practices permit teacher-stu-

dent-and-community interactions from either

recognition forms or contempt forms. As a re-

sult, it strengthens or harms the political and

social subject as to his/her self-respect and

self-steem with implications for playing social

and political roles within his/her context.

Key words: education, civic education, social

justice, recognition theory. comunidad de pertenencia básica en la sociedad; para ello, se apoya en el proceso de formación del ciudadano reflexivo, con autonomía, con conciencia de pertenencia y con valor social, con responsabilidades y derechos para participar en las decisiones en una sociedad justa y democrática (Naval, 2002). La educación apoyada en la Pedagogía y en la Didáctica de los diferentes saberes de la ciencia favorece las condiciones necesarias para esta formación ciudadana de manera integral (Díaz y Quiroz, 2005), impactando positivamente el desarrollo de todas las dimensiones del ser ciudadano -física, psicológica, social, individual, comunitaria, espiritual, política, entre otras- asumido desde lo individual y desde lo colectivo, facultado para ejercer su ciudadanía adecuadamente.

Los avances en la investigación sobre la formación ciudadana cobran importancia por cuenta del abordaje de asuntos como la igualdad de oportunidades, la pobreza, los derechos laborales, sindicales y de las minorias de toda clase (étnica, religiosa, lingüistica, nacional, sexual), se trata de necesidades cada vez más demandantes en los procesos de formación de sujetos sociales y políticos y en la construcción de las naciones. Así, el resultado de una formación ciudadana favorece el ejercicio de la ciudadanía consciente, solidaria, democrática y justa. A este respecto se tiene que las teorias ciudadanas permiten reflexionar desde la formación ciudadana sobre la mirada que se tiene en la educación, en términos de justicia social, desde las tres tradiciones propuestas por Marshall (1949): Liberalismo, Republicanismo y Comunitarismo, el cual avizora al ciudadano en una comunidad política con libertades individuales (derechos civiles), con una participación colectiva (derechos políticos) y en donde este ciudadano goza de las condiciones básicas para el desarrollo de una vida digna, con trabajo, educación, salud (derechos sociales). Mientras que una visión comunitarista reconoce a los ciudadanos desde comunidades concretas, incorporando la diferenciación entre grupos oprimidos y excluidos, el liberalismo como tradición del pensamiento ha hecho fuerza en el valor de la libertad, en el que el Estado debe velar por el fortalecimiento de las condiciones materiales e institucionales -la educación, una de ellas- para que cada individuo alcance su idea de vida feliz. A este respecto, Honneth plantea que se deben garantizar las condiciones sociales necesarias para que cada persona se realice dentro de su idea de vida feliz, mediado por las esferas de comu- 


\footnotetext{
${ }^{3}$ Blackwell, Cambrige Journals Online, Dialnet, Doaj, Eric, Jstor, Oecd, Oxford Journals, Redalyc, Science Direct, Springer Link y SciELO.
}

nicación social -familia, Estado y sociedad civil-, lo cual permitiría un reconocimiento reciproco - desde el amor, el derecho y la solidaridad-por medio de un mayor poder de acción entre los miembros de una sociedad concreta.

Es en este marco dentro del cual emerge la necesidad de comprender las relaciones entre la formación ciudadana y la justicia social en la educación desde la Teoría del Reconocimiento, analizando para ello asuntos como: la redistribución (económica), el reconocimiento (cultural) y la representación (política) (Fraser y Honneth, 2006); la igualdad de posiciones y oportunidades (Dubet, 2005); la heterogeneidad y la subjetividad desde Young (1990), quien plantea una política de la diferencia en oposición a las miradas de la justicia solo en cuanto a la distribución, analizando los mecanismos que utiliza el sistema de la dominación y la opresión; la justicia desde las capacidades humanas (Nussbaum, 2012); los derechos de las clases sociales más allá de la distribución de la riqueza (Walzer, 1993); la importancia de la participación de los ciudadanos (Miller, 1999) y sobre la justicia, desde la Teoría del Reconocimiento (Honneth, 1997; Ricoeur, 2006). Es de este punto del que se parte para analizar las relaciones existentes entre formación ciudadana y justicia social en la educación, campos en los que la redistribución y el reconocimiento de los derechos son urgentes frente a las inequidades sociales que en ella se evidencian. Se trata, específicamente, de un diálogo deconstructivo que integre y trascienda hacia nuevos lenguajes de la formación ciudadana y la justicia social en la educación, en el que los diferentes agentes educativos participen desde los discursos, las mutuas acciones y las relaciones frente a las prácticas sociales. Es importante resaltar que la formación ciudadana desde los años 90 es un área emergente en el ámbito académico (Quiroz, 2011), el mismo que está interesado en comprender mejor este concepto desde las relaciones que plantea con la justicia social en la educación desde la Teoría del Reconocimiento, debido a que en la mayoria de las producciones rastreadas se encuentra un vacío conceptual en esta temática, que no permite develar la fluidez y las tensiones entre ellos.

En el ejercicio de antecedentes, al rastrear en la literatura las investigaciones identificando las relaciones pretendidas, se utilizaron doce bases de datos ${ }^{3}$. La mayoría de los artículos de investigación revisados está relacionada con la dimensión jurídica y hace énfasis en las organizaciones comunitarias, los líderes 
De Prácticas y discursos

Universidad Nacional del Nordeste

Centro de Estudios Sociales

Año 8, Número 11, 2019, 0ctubre

ISSN 2250-6942

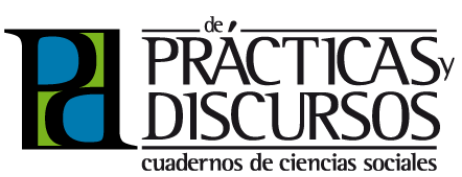

sociales, las oportunidades de los diferentes actores sociales, los derechos sindicales, la discriminación social y la búsqueda por el respeto de los derechos humanos. En los estudios encontrados sobre formación ciudadana se resaltan los balances historiográficos, conjugados con reflexiones sobre la responsabilidad social. Los aportes en el ámbito de la formación ciudadana se asumen desde diferentes perspectivas: Formación Integral (Díaz y Quiroz, 2005); La biblioteca pública, lugar para la construcción de ciudadanía (Jaramillo, 2011); Entre la educación para la ciudadanía y la formación ciudadana en la universidad (Mesa, 2011); La formación ciudadana dinamizada desde la motricidad (Benjumea, 2012); La formación ciudadana en perspectiva del potencial pedagógico de los estudios del territorio: Ciudadano territorial (Gutiérrez, 2012); Análisis de la triada Formación ciudadanaProyecto Político-Territorio (Pimienta, 2012); Formación ciudadana: ¡Utopía posible! (Gutiérrez y Pulgarín, 2009), entre otros.

En cuanto a la justicia social en la educación, al referirse a la necesidad de buscar una distribución de los recursos, trata la diferencia como posibilidad para la búsqueda de los derechos sociales y permite un giro en la mirada, introduciendo los principios de libertad e igualdad en la idea de justicia como equidad (Nussbaum, 2012) del reconocimiento de los derechos de las clases sociales más allá de la distribución de la riqueza (Fraser y Honneth, 2006) y de la participación (Miller, 1999; Young, 1990), para instaurarse en el desarrollo de las sociedades que mejoren las condiciones de vida de los ciudadanos. Sobresalen en los artículos dos tendencias principales: la primera resalta los retos que tiene el sistema educativo en Latinoamérica (Briceño, 2002; Magendzo, 2012) y la necesidad de ampliar la mirada hacia los problemas educativos desde las políticas educativas (Tello, 2011); la segunda se enfoca en la escuela como uno de los espacios donde se pueden leer los niveles de justicia social existentes en la sociedad (Connell, 2006), subrayando la importancia de la justicia en algunos agentes educativos (Pagès y Oller, 2007; Alucín, 2013). En este tema, la revista Sage Journals Education, citizenship and social justice desde 2006 facilita un foro valioso para el diálogo internacional y multidisciplinario, donde académicos y dirigentes de políticas públicas interactúan sobre los asuntos de la ciudadanía y la justicia social en la educación. En el contexto anglosajón son destacables, por su volumen y calidad, los trabajos académicos que integran siete manuales (handbooks) que 
recopilan artículos escritos por especialistas de la justicia social en la educación; estos manuales principalmente se reúnen en tres títulos, cada uno de ellos con diferentes ediciones asi: Teaching for diversity and social justice cuenta con tres ediciones (1997, 2007 y 2015); allí el énfasis se hace en los fundamentos y principios teóricos de la práctica de la justicia social, además de los principios del diseño de la práctica como educadores de la justicia social. También su atención se centra en los diseños curriculares para la diversidad y la justicia social. Readings for diversity and social justice tiene de igual forma tres ediciones (2000, 2010, 2013); el acento en esta ocasión se pone en la "diversidad social", subrayando las categorias sociales de raza, género, sexo, sexualidad y clase, y Handbook of Social Justice in Education (2009) enfatiza en tres pilares o principios de la justicia social en la educación: la equidad, el activismo y la alfabetización social a partir de los siguientes tópicos: perspectivas históricas y teóricas; perspectivas internacionales sobre justicia social en la educación; raza, etnicidad e idioma: buscando la justicia social en la educación; género, sexualidad y justicia social en la educación; cuerpos, juventud, discapacidad y la lucha por la justicia social en la educación.

En los handbooks se nota un marcado énfasis en las últimas ediciones en asuntos curriculares y didácticos, con la propuesta de contenidos que en el aula pueden tratarse o hacen referencia a la justicia social, con lo cual sirven como base para orientarse en las producciones y los intereses sobre la justicia social en la educación en nuestro contexto. En Colombia, este campo comienza a configurarse como referencia para la formación ciudadana, donde es primordial comenzar una reflexión sobre percepciones y el estado de la justicia social en la educación en el sistema educativo, para luego trazar rutas de intervención desde diferentes frentes y sujetos, pasando por lo epistemológico, pedagógico, didáctico y curricular, todo en atención al contexto político que se vive.

Para nuestro contexto colombiano, Rengifo (2013) resalta la importancia del reconocimiento recíproco como principal motor de la formación ciudadana. En este mismo contexto se encuentran producciones que, aunque no mencionan literalmente estas relaciones entre formación ciudadana y justicia social en la educación, si abordan el vínculo entre formación ciudadana y educación, donde, además de exponer situaciones epistemológicas y didácticas (González, 2012), se resalta el reconocimiento de la ciudadanía 
De Prácticas y discursos

Universidad Nacional del Nordeste

Centro de Estudios Sociales

Año 8, Número 11, 2019, 0ctubre

ISSN 2250-6942

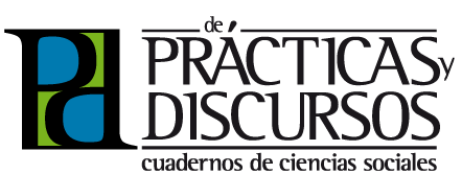

en la perspectiva de derechos (Alvarado y Carreño, 2007).

De esta manera, el estado de este tema reflejado en la revisión de esta literatura deja en evidencia que en las definiciones sobre el concepto de justicia rondan extremos, discontinuidades, simultaneidades y recurrencias; sin embargo, lo que ha posibilitado estos avances son las percepciones, vivencias y descripciones sobre las injusticias. Algunos autores han privilegiado este análisis deconstructivo del concepto (Margalit, 1996) debido a que no hay consenso en su definición. Su base filológica desentraña posturas en cuanto a tiempo, modo y lugar de producción de la idea de la justicia social; lo cual permite tomar como punto conceptual los avances epistemológicos de la Escuela de Frankfurt sobre la justicia social desde la Teoria del Reconocimiento, permitiendo desentrañar las relaciones y tensiones con la formación ciudadana.

\section{TEORÍA DEL RECONOCIMIENTO}

Honneth (1997) analiza el papel de la lucha por el reconocimiento en las esferas del amor, el derecho y la solidaridad -modos de reconocimiento- ya que existen formas de menosprecio que corresponden a experiencias negativas que hacen daño en las principales instituciones que vivencia el ser humano como son la familia, el Estado y la sociedad civil-esferas de comunicación. La formación del "yo justo" se realiza desde la interacción con los otros y el reconocimiento mutuo de las esferas en la familia, la sociedad civil y el Estado, donde el enfrentamiento con su identidad complementado por los otros sujetos permite formar la autonomía. Sin embargo, estas relaciones de reconocimiento, desde el amor, el derecho y la sociedad civil, se evidencian a partir de la representación del conflicto como un mecanismo de colectivización social.

La propuesta de Honneth sobre el reconocimiento va más allá de la distribución individual y económica; se ocupa, por el contrario, del aseguramiento de las esferas de comunicación -la familia, el Estado y la sociedad civil- donde las personas se mueven cotidianamente y desempeñan un papel primordial para el afianzamiento de la autonomía, el autorrespeto, la autoestima, el autoconcepto y el autocuidado -autorealizaciones prácticas-. En ese orden, las relaciones que se establecen con la familia, los amigos, el trabajo y la sociedad posibilitan una visión de la justicia social, 
según las retribuciones que el individuo reciba en términos de oportunidades de sus cualidades en estas relaciones sociales. Esta concepción de pensamiento cuenta con aportes de la psicología social desde el argumento de que "el ser humano le debe su identidad a la experiencia de un reconocimiento intersubjetivo" (Honneth, 1997: 90), donde la lucha por el reconocimiento es la referencia para explicar el desarrollo moral de la sociedad; es en la interacción con los otros donde se logra el reconocimiento de sí mismos, pues nos permite leer las percepciones que tendriamos sobre nuestra propia vida. Honneth parte de este reconocimiento del "yo" desde "el otro" para ampliar el rango de expansión de "lo otro", permitiendo que también se ensanche el circulo de referencia de la autoimagen práctica.

Es en los procesos de formación social, en la familia, el Estado y la sociedad civil, donde los sujetos adquieren sus primeras experiencias de la justicia/injusticia por medio de relaciones negativas o positivas, lo cual no solo permite un empuje normativo social, sino que forma el desarrollo del derecho en su conformación interna. La idea principal de Honneth es que el reconocimiento de una persona no solo se obtiene en las esferas públicas donde el sujeto se reconoce como portador de derechos, sino que esta introyección se adquiere desde nuestras primeras vivencias sociales en la esfera intima, por ejemplo, desde nuestras primarias relaciones de amor, de afecto, cuidado, con vínculos desde la familia y los amigos, marcando nuestra percepción sobre el entorno y sobre nosotros mismos. Específicamente, propone a partir del modelo hegeliano tres formas de reconocimiento, ligadas a formas de menosprecio:

\section{Tabla1: Esferas del reconocimiento social de Axel Honneth}

\begin{tabular}{|l|l|l|l|}
\hline \multicolumn{2}{|l|}{ Estructura de las relaciones de reconocimiento social } \\
\hline $\begin{array}{l}\text { Modos de } \\
\text { reconocimiento }\end{array}$ & $\begin{array}{l}\text { Dedicación } \\
\text { emocional }\end{array}$ & $\begin{array}{l}\text { Atención } \\
\text { cognitiva }\end{array}$ & \multicolumn{1}{|l|}{$\begin{array}{l}\text { Valoración } \\
\text { Social }\end{array}$} \\
\hline $\begin{array}{l}\text { Dimensión de } \\
\text { personalidad }\end{array}$ & $\begin{array}{l}\text { Naturaleza de la } \\
\text { necesidad y del } \\
\text { afecto }\end{array}$ & $\begin{array}{l}\text { Responsabilidad } \\
\text { moral }\end{array}$ & $\begin{array}{l}\text { Cualidades y } \\
\text { capacidades }\end{array}$ \\
\hline $\begin{array}{l}\text { Formas de } \\
\text { reconocimiento }\end{array}$ & $\begin{array}{l}\text { Relaciones primarias } \\
\text { (amistad y amor) }\end{array}$ & $\begin{array}{l}\text { Relaciones de derecho } \\
\text { (derechos) }\end{array}$ & $\begin{array}{l}\text { Comunidad de valor } \\
\text { (solidaridad) }\end{array}$ \\
\hline
\end{tabular}


De Prácticas y discursos

Universidad Nacional del Nordeste

Centro de Estudios Sociales

Año 8, Número 11, 2019, Octubre

ISSN 2250-6942

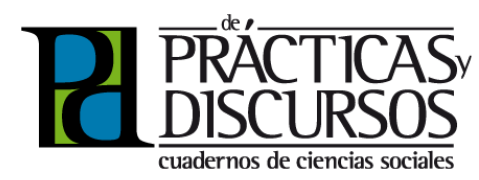

\begin{tabular}{|l|l|l|l|}
\hline $\begin{array}{l}\text { Potencial de } \\
\text { desarrollo }\end{array}$ & $\begin{array}{l}\text { Generalización y } \\
\text { materialización }\end{array}$ & $\begin{array}{l}\text { Individualización e } \\
\text { igualación }\end{array}$ \\
\hline $\begin{array}{l}\text { Autorrelación } \\
\text { práctica }\end{array}$ & Autoconfianza & Autorespeto & Autoestima \\
\hline $\begin{array}{l}\text { Formas de } \\
\text { menosprecio }\end{array}$ & $\begin{array}{l}\text { Maltrato y violación, } \\
\text { integridad física }\end{array}$ & $\begin{array}{l}\text { Desposeción de } \\
\text { derechos y exclusión; } \\
\text { integridad social }\end{array}$ & $\begin{array}{l}\text { Indignidad e injuria, } \\
\text { "honor", dignidad }\end{array}$ \\
\hline
\end{tabular}

FUENTE: Honneth, 1997: 159.

Los modos de reconocimiento están unidos a la autorrealización práctica por medio de tres esferas de comunicación: la familia desde la forma del amor, el Estado desde la forma del derecho y la sociedad civil desde el trabajo a partir de la solidaridad. A su vez, la negación de estas formas de reconocimiento en cada uno de los niveles propuestos conlleva formas de menosprecio, las cuales "desempeñan para la integridad psíquica del hombre el mismo papel negativo que las enfermedades en la reproducción de su cuerpo: por la experiencia de la desvalorización de la humillación social, los seres humanos peligran en su identidad" (Honneth, 1997: 164).

Las experiencias de menosprecio, que en Hegel se abordan como principios del "delito", se interconectan con el comportamiento destructivo desde las experiencias incompletas del reconocimiento en cada una de las esferas. Estas formas de menosprecio van desde el maltrato físico hasta la injuria, en las que la humillación convierte lo personal en un asunto englobante que lleva a la destrucción de la autorreferencia, no solo del cuerpo, sino de la psiquis del ser humano, al punto de llevarlo a la indefensión frente al agresor arrebatándole la percepción de su realidad y su valoración social.

\section{JUSTICIA SOCIAL DESDE EL RECONOCIMIENTO}

Desde esta perspectiva, la mirada de la justicia desde la Teoria del Reconocimiento permite el análisis de miradas hegemónicas de la justicia liberal, donde su acento está en las libertades y autonomía individual; ampliando la propuesta de la justicia no solo a la distribución de los recursos económicos individuales, sino entendiendo que una "distribución justa debe ocuparse del aseguramiento de aquellas esferas de la comunicación social que hacen posible el reconocimiento recíproco" (Cortés, 2007: 95). La 
propuesta de Honneth va más allá de la distribución individual y económica, se ocupa, por el contrario, del aseguramiento de las esferas de comunicación social -familia, Estado y sociedad civil- orientadas a las condiciones sociales que hacen viable el alcance de los individuos de su autorrealización en términos de la autonomía, el autorrespeto y la autoestima. Las relaciones que se establecen con la familia, los amigos, el trabajo y la sociedad posibilitan una visión de la justicia social, según las retribuciones que el individuo reciba en términos de oportunidades de sus cualidades en estas relaciones sociales.

Con respecto a la posición de Honneth, el giro cultural que se vivencia hoy no se encuentra solo en las luchas por la distribución de los recursos, sino en las cuestiones básicas de nuestra forma de vida, donde el reconocimiento de las convicciones colectivas e individuales deben estar centradas en las experiencias de sufrimiento que experimentan los sujetos en su vida cotidiana, superando las visiones que solo abarcan la esfera política pública, trascendiendo a los fenómenos de privación social que no han sido evaluados por la tradición liberal de la justicia. Por ejemplo, el aislamiento social, los desórdenes en la vida privada, la descalificación de las destrezas, los fenómenos de empobrecimiento masivo y las privaciones de las comunidades oprimidas por el sistema hegemónico.

\section{LUCHA POR EL RECONOCIMIENTO}

En la investigación que evidenció las relaciones entre la formación ciudadana y la justicia social en la educación nos centramos en la visión de la justicia social desde la Teoría del Reconocimiento, debido a que analiza la construcción social no solo desde aspectos capitalistas de base, sino que, por el contrario, resalta los procesos de formación social en esferas como la familia, el Estado y la sociedad civil, donde los sujetos adquieren sus primeras experiencias de la justicia/injusticia, por medio de relaciones negativas o positivas, lo cual no solo permite un empuje normativo social, sino que forma el desarrollo del derecho en su conformación interna.

Los modos de reconocimiento -amor, derecho y solidaridadestán unidos a la autorrealización práctica -autoconfianza, autorrespeto, autoestima- por medio de tres esferas de comunicación: la familia desde la forma del amor, el derecho con el 
De Prácticas y discursos

Universidad Nacional del Nordeste

Centro de Estudios Sociales

Año 8, Número 11, 2019, Octubre

ISSN 2250-6942

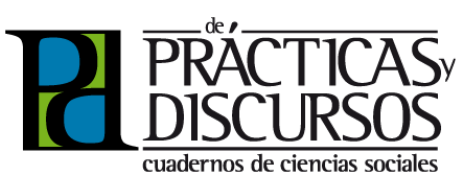

Estado y el trabajo a partir de la solidaridad. A su vez, la negación de estas formas de reconocimiento en cada uno de los niveles propuestos en la anterior conlleva a formas de menosprecio. Es evidente que, con experiencias de menosprecio, se sufre una lesión que cambia totalmente la forma de reconocimiento de un sujeto, esto dependiendo de la esfera afectada y la profundidad de los daños o trastornos que se producen. Estas formas de menosprecio van desde el maltrato físico hasta la injuria; donde la humillación, entendida esta desde las miradas comunitaristas como "exclusión de la comunidad humana. Humillación, degradación, desprecio no-reconocimiento" (Savarwald, 2008:30). A continuación, se exponen las caracteristicas de cada una de las esferas del reconocimiento con sus correspondientes formas del menosprecio: amor (maltrato, violación), derecho (exclusión, desposesión) y solidaridad (indignación, injuria).

Amor. Para la Teoría del Reconocimiento, en el sentimiento experimentado del ser amado, apreciado, cuidado y querido, es el primer acercamiento natural de los sujetos para confirmar su individualidad. Cuando se habla de las relaciones, se hace referencia a "todas las relaciones primarias, en la medida en que, a ejemplo de las relaciones eróticas entre dos, las amistades 0 las relaciones padres-hijos estriban en fuertes lazos afectivos" (Honneth, 1997: 117). Por tanto, estos vínculos son la base para la formación de la identidad personal de los sujetos, a partir de determinados actos de reconocimiento. Respecto a la relación padres e hijos, el amor representa el primer estadio de reconocimiento reciproco, en esa necesidad de cuidado y atención que un recién nacido requiere; los sujetos se sienten unificados, la dependencia tácita existe desde el nacimiento y se hace expansiva a los primeros años de vida. Sin embargo, este sentimiento de unicidad debe ser reemplazado por el de autonomía, de manera que su resultado debe ser la "supresión (Aufhebung) de aquella unificación del sentimiento"" (Honneth, 1997: 30).

El reconocimiento pleno a partir de unas condiciones favorables emocionales permite la formación del autorrespeto y el autoconcepto, lo cual desemboca a futuro en un sujeto capaz de afrontar la vida pública; es decir, de las primeras percepciones que se tienen del yo depende su desenvolvimiento autónomo en los espacios políticos por fuera de la esfera intima (Honneth, 1997), en el cual se sienta con dignidad de vida, con posibilidades de derechos y deberes. 
Estas relaciones amorosas constituyen una forma de reconocimiento en la que la formación de la identidad personal lleva a la autoestima, al autocuidado, asunto vital para la constitución del sujeto político. He aquí una primera unión de las tres esferas de reconocimiento. ¿Qué pasa, entonces, cuando estas relaciones amorosas no están ligadas a sentimientos de cuidado, atención y respeto, sino que, por el contrario, priman el maltrato y las lesiones en estas relaciones desde lo intimo? En este sentido, prima la pérdida de la autoconfianza, se destruyen los perfiles primordiales de autorreferencia, experimentando por medio del menosprecio de los seres que pretendían solo sentimientos positivos una especie de venganza social hacia el mundo que lo circunda (Fraser y Honneth, 2006). La esfera del amor cobra importancia para la vida en comunidad, por los sentimientos que actúan como motores para los movimientos involuntarios que realizan los sujetos con los otros en su vida pública; las relaciones primarias, sin duda, son base fundamental para generar emociones de simpatía y atracción que determinen la esfera del derecho.

Derecho. Como segunda esfera de reconocimiento, este posibilita a través del Estado una valoración de cercanía social con los demás miembros de la comunidad, donde la base intersubjetiva parte de tratar a los demás como a sí mismo. El "nosotros" fusionado en la voluntad del todos genera un grado de conciencia que crea relaciones de valoración social, lo cual converge en los acuerdos sociales que la voluntad general denomina normas, cuyo cumplimiento se garantiza y obliga a los sujetos al respeto de los miembros de la comunidad, donde cada uno puede tomar una posición positiva de sí mismo, convirtiéndose en responsable de sus propios actos en la esfera del reconocimiento social. El respeto de sí es vital en esta nueva esfera, pues trasciende a la comunidad como signo de respeto social, en el que el reconocimiento de los derechos individuales no es visto como simples islas, sino como la garantía de considerar a los sujetos como seres libres, capaces de tomar decisiones responsables desde la capacidad de generar juicios autónomos de la realidad vivida (Honneth, 1997).

Algo destacable en esta esfera es cómo el ciudadano se orienta al respecto de normas morales y cómo llega a ser reconocido jurídicamente o no. Este reconocimiento jurídico es ampliado a las relaciones sociales debido a la expansión de las vivencias prácticas que experimentan los sujetos, lo que produce que sienta identificación de respecto de los otros, dependiendo del hecho 
De Prácticas y discursos

Universidad Nacional del Nordeste

Centro de Estudios Sociales

Año 8, Número 11, 2019, Octubre

ISSN 2250-6942

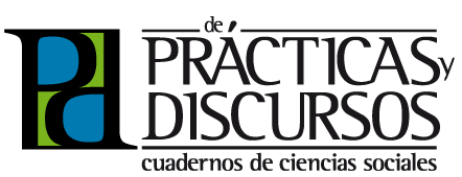

que les acontece o la vivencia positiva que se presente en la esfera pública. La lucha social, según Hegel (2006), surge de las formas de menosprecio identificadas en la esfera pública, en las que los sujetos de derecho han vivenciado desposesión de derechos o exclusión social, manifestados no solo en la privación de la autonomía personal, sino en la condición de no sentirse como un sujeto valioso o plenamente igual en la interacción jurídica (Honneth, 2011). En tal sentido, cuando se le priva de sus derechos, se lesionan sus expectativas de ser reconocido, truncando su capacidad de juicio moral, donde esta experiencia va acompañada de la pérdida del respeto de sí mismo y de su capacidad de interacción con los demás sujetos como iguales.

Solidaridad. Como tercera esfera de reconocimiento se da en la sociedad donde los sujetos comparten con los otros; se convierte en un espacio coaccionante en el que la valoración social posibilita diferenciar las condiciones y los aportes de cada ciudadano para la realización de los objetivos sociales (Honneth, 1997). Los valores fijados socialmente son el punto de partida para analizar la cooperación que cada uno de sus participantes realiza para lograr "una comunidad valorativa por la orientación a objetivos comunes" (Honneth, 1997: 150).

Otra variante importante son los cambios que se producen con el paso del tiempo en los objetos éticos de una sociedad; por esto, no se puede pensar en la justicia como un concepto ad hoc o inmóvil. Por el contrario, es contextual (espacio-tiempo) y está directamente relacionada con los acuerdos que cada comunidad pacte como aspectos positivos para su desarrollo o para su idea de vida feliz. Como sujetos sociales, nos consideramos valiosos cuando las percepciones de los otros sobre nuestras cualidades y capacidades se tornan útiles y diferenciadas, convirtiéndose en posibilidades para el desarrollo positivo del colectivo en el cual me desenvuelvo. Se puede afirmar que las bondades singulares que se aportan a cada comunidad de pertenencia permiten la adquisición de un prestigio que, en perspectiva, contribuye a la autorrealización personal. Sentirse perteneciente a un grupo, valorado y necesitado por el mismo, lleva a un sentimiento de orgullo colectivo, en el que se lucha por llevar a cabo operaciones conjuntas para la consolidación y permanencia de esta unidad social.

Las interacciones dentro de cada grupo, donde cada uno apoya y defiende las vidas participantes y diferenciadas en forma proporcional, implican el uso de la solidaridad. Para Honneth (1997), 
esta solidaridad surge cuando el grupo lucha por su modo de vida, en resistencia a la opresión política, en la que cada uno se identifica a si mismo y reconoce a los otros. Si continuamos naturalizando por décadas ideas frente al racismo, la inmigración, el sexismo, las diferencias religiosas o la violencia, estaremos generando inequidades.

\section{RELACIONES ENTRE FORMACIÓN CIUDADANA Y JUSTICIA SOCIAL EN LA EDUCACIÓN DESDE LA NARRATIVA DE LOS LÍDERES DE COLEGIOS MAESTROS}

Los discursos contenidos en las narrativas de los líderes sobre las prácticas de formación ciudadana y justicia social en la educación, desde la Teoría del Reconocimiento, fueron las fuentes de las cuales brotaron las relaciones entre estos conceptos. Estas narrativas tomaron forma y contenido en las trayectorias espacio-temporales de memoria, experiencia presente y expectativa futura; se notó en las narrativas de los líderes, por un lado, que la memoria y la experiencia presente conviven, simultáneamente, para configurar la expectativa futura que se producen en y a través de las prácticas que acompañan las esferas del reconocimiento. Por otro lado, fue notorio que en las narrativas de los líderes cada esfera del reconocimiento, amor-derecho-solidaridad, adquiera una secuencialidad diferente a la expresada en la Teoría del Reconocimiento y asume a veces sentidos insospechados o cargas semánticas que ponen de relieve una esfera $u$ otra como condición de posibilidad o de existencia de las otras dos, y que la simetría, el equilibrio y la secuencialidad en las que se dan las esferas de reconocimiento se presenta solo en la Teoría del Reconocimiento de Axel Honneth: del amor al derecho y de este a la solidaridad, pero no como lo experimentaron y lo manifestaron los líderes del proyecto Colegios Maestros de Medellín. Se puso de manifiesto en la memoria de los líderes un asunto bastante llamativo que ubica a la esfera del derecho en la posición más destacada, nombrada y enfatizada en el diálogo narrativo, en tanto fue puesta como punto de partida y argumentación para definir todo aquello que los ha habitado y que los cuestiona, al punto de que, al mencionar aspectos y características de las demás esferas (amor, solidaridad), el marco de referencia siempre estaba ligado a referencias de derecho, bien por exaltación, oposición o reclamo de la coherencia, pertinencia 
De Prácticas y discursos

Universidad Nacional del Nordeste

Centro de Estudios Sociales

Año 8, Número 11, 2019, Octubre

ISSN 2250-6942

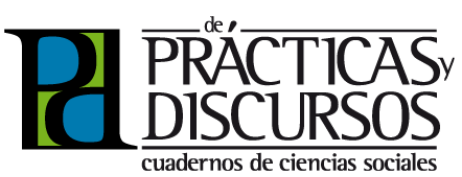

y sentido que ella misma ha cobrado y cobra en su existencia. En los siguientes apartados se exponen las intersecciones encontradas con las tres esferas del reconocimiento, haciendo énfasis especial en el derecho como punto de relacionamiento entre la formación ciudadana y la justicia social en la educación. Sin embargo, y a pesar de esta supremacía de la esfera del derecho, es evidente que cada narrativa se encuentra atravesada explícita o implícitamente por las otras dos esferas (amor-solidaridad) y aunque se narre desde una referencia temporal específica, los otros ejes de tiempo (memoria, experiencia presente, expectativa futura) tienen presencia en ellas.

\section{NARRANDO DESDE LAS INTERSECCIONES DE LA ESFERA DEL DERECHO}

La esfera del derecho se presenta en estas narraciones como el resultado del encuentro entre los sujetos, las instituciones y los discursos de una manera dialéctica que tiene la impronta de complejos procesos en los cuales la presencia de las formas del menosprecio no solo del derecho, sino también de la solidaridad y el amor, son constitutivas en los procesos de formación de la autoconfianza, el autorrespeto y la autoestima de los sujetos en los Colegios Maestros. Asunto que permite plantear nuevos aspectos a tener presente para el desarrollo de las instituciones, que garanticen condiciones justas y de reconocimiento no solo a nivel individual, sino que se extienda a los colectivos, en este caso privilegiando a los más vulnerables, desiguales y excluidos. En sintesis, puede decirse que la esfera del derecho en las narrativas de los líderes muestra varios aspectos a tener en cuenta: -Un primer aspecto es que cada narración cuenta con una intersección donde se observan las tres esferas del reconocimiento (amor, derecho y solidaridad) y los ejes de tiempo (memoria, experiencia presente, expectativa futura), haciendo énfasis en una de ellas desde lo narrado, pero que resaltan lo poroso de estas relaciones y las posibilidades de analizar el reconocimiento como un proceso dialéctico.

-Un segundo aspecto tiene que ver con que las formas y contenidos de lo narrado, ya que expresan una relación entre los procesos de la formación ciudadana y la justicia social en la educación que, al examinarse desde la Teoría del Reconocimiento, permiten entender que los discursos sobre las prácticas que alli se mani- 
fiestan son el resultado de inmanentes formas del menosprecio y aunque la tendencia más destacada en la esfera del derecho está relacionada con las formas del menosprecio, el resultado que se puede observar no es concomitante con la lógica de estas, en tanto los sujetos reiteradamente hacen de ellas una posibilidad para producir nuevos rumbos a las trayectorias imaginadas que en la lógica de las formas del menosprecio deberían producirse. - Un tercer aspecto alude a que cada narración interpela la particular manera en la que lo institucional ha instalado, el sentido de la formación ciudadana, una formación ciudadana de corte comunitarista que hace del sistema de relacionamiento entre las instituciones y los sujetos un proceso continuo, coherente y armonioso que es ajeno a las irrupciones de la contingencia y a las posibilidades de la transformación que impone la dialéctica misma de la existencia. En otras palabras, todos los líderes destacan sus comunidades de pertenencia, como impulsadoras y coequiperas para la superación de las adversidades o formas del menosprecio, convirtiéndose este factor en posibilidad para pensar en los grupos sociales que se conforman, donde la escuela como posibilidad de la formación ciudadana, en este caso, nos permite fortalecer las formas del reconocimiento recíproco.

\section{NARRANDO DESDE LAS INTERSECCIONES DE LA ESFERA DEL AMOR}

Con respecto a la esfera del amor, y desde la Teoría del Reconocimiento, las narraciones de los agentes educativos, en este caso los líderes del proyecto Colegios Maestros de Medellín, estuvieron centradas en varios tópicos: experiencias vividas en su niñez, relaciones establecidas con sus padres, vínculos con otros familiares y proyecciones de futuro desde los modelos ejemplarizantes. En síntesis, puede decirse que la esfera del amor en las narrativas de los líderes muestra:

-Que la relación entre los procesos de la formación ciudadana y la justicia social en la educación tienen la oportunidad de convertir formas del menosprecio en experiencias del reconocimiento por medio de acciones claras de directivos docentes y docentes, donde el acompañamiento en los procesos formativos y la generación de lazos fuertes con sus compañeros y toda la comunidad educativa pueden permitir el afianzamiento de la autoconfianza, además de ayudar desde lo pedagógico en la gene- 
De Prácticas y discursos

Universidad Nacional del Nordeste

Centro de Estudios Sociales

Año 8, Número 11, 2019, Octubre

ISSN 2250-6942

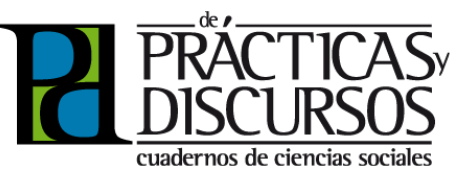

ración de procesos de autocuidado y de identificación de formas del menosprecio que muchas veces pueden ser superadas desde la esfera del derecho.

-Que las prácticas que acompañan esta esfera producen otro modo de ordenamiento de las formas y contenidos de lo social, requiriendo muchas veces la intervención del Estado para garantizar la preservación de la vida y de los derechos básicos de los ciudadanos. Este proceso puede ser direccionado desde la escuela, por ejemplo, con la identificación de entornos caracterizados por maltrato y violación de derechos, para ser intervenidos desde lo institucional.

-Que la esfera del amor se describe como una estructura plagada de múltiples cortes que llevan a que tanto sus formas de menosprecio, como las del derecho y la solidaridad, en intersección con los ejes de tiempo (memoria, experiencia presente y expectativa futura), sirvan como condición de posibilidad y de existencia de renovadas acciones mediante las cuales los sujetos reinventan lo que por naturaleza les fue dado y por imposición les fue heredado en nuevas formas y contenidos que creativamente llevan a convertir lo absurdo en trascendente. Lo anterior permite observar una recurrencia en las narraciones de los líderes, donde cada discurso evidencia la presencia en supremacía de una esfera del reconocimiento y un eje de tiempo, sin omitir la presencia de las otras esferas y ejes que, de forma explícita o implícita, hacen presencia en cada una de ellas.

\section{NARRANDO DESDE LAS INTERSECCIONES DE LA ESFERA DE LA SOLIDARIDAD}

En esta esfera del reconocimiento, los asuntos extensivos de los valores potenciados durante las etapas vivenciadas en instituciones como la familia y la escuela trascienden a la comunidad donde el sujeto aporta de manera sustancial su material intelectual, moral y social, permitiendo reconocimiento recíproco, lo cual genera valoración social, desde formas del reconocimiento como la consideración, el prestigio, el respeto social, cultural y la cooperación. En sintesis, puede decirse que la esfera de la solidaridad en las narrativas de los líderes muestra que:

-La esfera de la solidaridad posee una relación directa con los procesos de la formación ciudadana y la justicia social en educación, en tanto, por efecto directo o tangencial, la solidaridad reúne todos aquellos aspectos de los que abrevan muchos de los 
sentidos de una y otra. Es decir, aquí se presenta la solidaridad como un espacio de metamorfosis en el cual las formas del menosprecio pasan de ser excluyentes a ser el referente desde el cual se dan acciones de transformación que buscan su eliminación de la gramática existencial.

-En el reconocimiento con y desde el otro, la esfera de la solidaridad está habitada por continuas referencias no solo a sus formas, sino también a las formas de las esferas del amor y del derecho, en tanto permiten que puedan generarse dispositivos de acción mediante los cuales se crean múltiples sentidos para establecer las redes de intercambio que caracterizan a la solidaridad misma.

- Los discursos asociados a las prácticas de reconocimiento en la esfera de la solidaridad son polivalentes y diversos, pero con una caracteristica en particular que los articula, y es el sentido de reconfiguración de la fatalidad en perspectiva de potencia y no de obstáculo.

\section{ALGUNAS CONCLUSIONES}

Presentada esta relación entre la formación ciudadana y la justicia social en la educación, desde la Teoría del Reconocimiento de Honneth, es posible considerar que en la escuela colombiana en este contexto del posacuerdo se requieren propuestas didácticas desde las Ciencias Sociales que puedan trabajar estas relaciones para luego trazar rutas de intervención desde diferentes frentes y sujetos, pasando por lo epistemológico, lo pedagógico y lo curricular, todo en atención al contexto político que se vive, a los conflictos, procesos de paz y a la preservación de la integridad de la vida. El reto es grande, toda vez que la escuela ha de asumir la superación de falencias en cuanto al amor, que siendo responsabilidad de la familia, no se lograron resolver alli y el estudiante entra con este vacío afectivo a una dinámica y un contexto educativo en el que prima el derecho y, a su vez, es el apoyo del desarrollo de la esfera de la solidaridad, con radio de acción en la universidad y en el trabajo. Este trabajo hace parte de una vertiente de teoría crítica actual, hay necesidad de seguir ahondando en trabajos de este tipo que ayuden a esclarecer más estas relaciones y posibiliten nuevos aprendizajes en este campo de investigación educativa.

ALUCíN, S. (2013). Ciudadanía y justicia social: Una mirada 
etnográfica sobre la educación secundaria en Argentina. Revista Internacional de Educación para la Justicia social 2(1), 4968. Recuperado de: http://www.rinace.net/riejs/numeros/vol2num1/art3.pdf

ALVARADO, S. Y CARREÑO, M. (2007). La formación ciudadana: una estrategia para la construcción de justicia. Revista Latinoamericana de ciencias sociales, niñez y juventud, (1-16).

BENJUMEA, M. (2012). La Formación Ciudadana dinamizada desde la Motricidad como Campo de configuración de lo humano: una lectura en escenarios y prácticas de la Recreación y el Deporte. (Tesis doctoral inédita). Facultad de Educación, Universidad de Antioquia. Medellín-Colombia.

BRICEÑO, R. (COMP.) (2002). Violencia, sociedad y justicia en América Latina. Buenos Aires: Clacso.

CONNELL, R. (2006). Escuelas y justicia social. Madrid: Ediciones Morata.

CORTÉS, F. (2007). Justicia y exclusión. Instituto de Filosofía de la Universidad de Antioquia. Medellín: Siglo del Hombre Editores.

DÍAZ, A. Y QUIROZ, P. (2005). Educación, instrucción y desarrollo. Medellín: Imprenta Universidad de Antioquia.

DUBET, F. (2011). Repensar la justicia social. Buenos Aires: Siglo XXI.

FRASER, N. Y HONNETH, A. (2006). Redistribución o reconocimiento. Madrid: Ediciones Morata.

GONZÁLEZ, G. (2012). La formación inicial del profesorado de ciencias sociales y la educación para la ciudadanía en Colombia: representaciones sociales y prácticas de enseñanza. (Tesis doctoral inédita). España: Universidad Autónoma de Barcelona.

GUTIÉRREZ, A. (2012). Formación ciudadana en perspectiva del potencial pedagógico de los estudios del territorio. Ciudadano territorial: fundamento de la democracia. (Tesis doctoral 
inédita). Facultad de Educación, Universidad de Antioquia. Medellín-Colombia.

HEGEL. G. (2006). Fenomenología del espíritu. España: Editorial Pre-textos.

HONNETH, A. (1997). La lucha por el reconocimiento: Por una gramática moral de los conflictos sociales. Barcelona: Editorial Crítica.

(2006). Redistribución como reconocimiento: Respuesta a Nancy Fraser. En Fraser, N. y Honneth, A. (ed.) Redistribución o Reconocimiento (pp. 89-148). Madrid: Ediciones Morata.

JARAMILLO, 0. (2011). La biblioteca pública, lugar para la construcción de ciudadania: una mirada desde la educación social. (Tesis doctoral inédita). Facultad de Educación, Universidad de Antioquia. Medellín-Colombia.

KYMLICKA, W. Y NORMAN, W. (1994). El retorno del ciudadano: Una revisión de la producción reciente en teoría de la ciudadanía. Cuadernos del CLAEH, (75), 81-112.

MAGENDZO, A. (2012). La educación en derechos humanos y la justicia social en educación. Santiago de Chile: Universidad Academia de Humanismo Cristiano.

MARGALIT, A. (1996). La sociedad decente. Estados Unidos: Harvard University Press.

MARSHALL, T.H. (1949). Ciudadanía y clase social: y otros ensayos. Cambridge: University Press.

MESA, A. (2011). El juicio de los espectadores de Hannah Arendt. Entre la Educación para la ciudadanía y la formación ciudadana en la universidad. (Tesis doctoral inédita). Facultad de Educación, Universidad de Antioquia. Medellín-Colombia.

NAVAL, C. (2002). Educar ciudadanos. La polémica liberalcomunitarista en educación. Pamplona: Ediciones Universidad de Navarra SA. 
NUSSBAUM, M. (2012). Las fronteras de la Justicia: Consideraciones sobre la exclusión. Madrid: Editorial Paidós.

PAGĖS, J. Y OLLER, M. (2007). Las representaciones sociales del derecho, la justicia y la ley de un grupo de adolescentes catalanes de $4^{\circ}$ de ESO. Enseñanza de las Ciencias Sociales, (6), 3-19.

PIMIENTA, A. (2012). Formación ciudadana, proyecto político y territorio. (Tesis). Medellín: Universidad de Antioquia.

TAYLOR, CH. (1993). El multiculturalismo y la "política del reconocimiento. México, DF: Fondo de Cultura Económica.

QUIROZ, R. (2011). Relaciones entre cultura política, tendencias pedagógicas y enseñanza de las ciencias sociales: un área emergente. En Formación ciudadana: una mirada desde Colombia y México. Universidad de Colima, Universidad de Antioquia.

RENGIFO, T. (2013). Formas de reconocimiento y formación ciudadana. (Tesis doctoral inédita). Ibagué: Universidad del Tolima.

RICOEUR, P. (2006). Caminos del reconocimiento. Tres estudios. México: Fondo de Cultura Económica.

SAVARWALD, G. (2008). Reconocimiento y liberación: Axel Honneth y el pensamiento latinoamericano. Berlín: Editorial LIT Verlag.

TELLO, C. (2011). Epistemologías de la política educativa y justicia social en América Latina. Nómadas. Revista Crítica de Ciencias Sociales y Jurídicas. MT (2011.1) Monográfico América Latina EMUI Euro-Mediterranean University Institute. Universidad Complutense de Madrid.

WALZER, M. (1993). Las esferas de la justicia. México: Fondo de Cultura Económica.

YOUNG, I.M. (1990). Justice and the politics of difference. Princeton, NJ: Princeton University Press. 